\title{
HST Observations Reveal the Curious Geometry of Circumgalactic Gas
}

\author{
Glenn G. Kacprzak ${ }^{1}$, Sowgat Muzahid ${ }^{2}$, Christopher W. Churchill ${ }^{3}$, \\ Nikole M. Nielsen ${ }^{1}$ and Jane C. Charlton ${ }^{2}$ \\ ${ }^{1}$ Swinburne University of Technology, Victoria 3122, Australia, \\ email: gkacprzak@astro.swin.edu.au \\ ${ }^{2}$ The Pennsylvania State University, State College, PA 16801, USA \\ ${ }^{3}$ New Mexico State University, Las Cruces, NM 88003, USA
}

\begin{abstract}
We have discovered that warm gas flows along galaxy major and minor axes detected out to $200 \mathrm{kpc}$. Our results are derived from a sample of HST-imaged isolated galaxies with nearby background quasars used to probe their $10^{5} \mathrm{~K}$ CGM detected in HST/COS UV spectra (traced by $\mathrm{O}$ VI absorption). We constrain the geometry of the gas to reside between 20-40 degrees of the projected major axis and within 60 degrees of the projected minor axis, with little-to-no gas found in between. Furthermore, strong absorption systems tend to be found along the minor axes of star-forming galaxies. All of our results are consistent with the current view of the CGM originating from major axis-fed inflows/recycled gas and from minor axis-driven outflows.
\end{abstract}

Keywords. quasars: absorption lines, galaxies: halos, galaxies: evolution

\section{Introduction}

The circumgalactic medium (CGM) supplies the gas reservoirs required for galaxy star formation and is replenished via accretion/recycling and outflowing gas. Determining how the CGM interacts with galaxies is critical to understanding how galaxies evolve. Models and observations indicate that gas accretion should occur along filaments co-planar to the galaxy disk, whereas outflows should extend along the galaxy minor axis.

Kacprzak et al. (2012) reported a bimodality in the azimuthal angle distribution of gas around galaxies, where cool dense CGM gas (traced by Mg II absorption) exists primarily along the projected galaxy major and minor axes (also see Bouché et al. 2012; Bordoloi et al. 2011; Lan et al. 2014). This is consistent with galaxy evolution scenarios where galaxies accrete new co-planar gas, forming new stars and producing metal-enriched galactic outflows. These conclusions are drawn from observations conducted using $\mathrm{Mg}$ II absorption, however both outflowing and infalling gas are expected to be multi-phased.

Although OVI absorption has been extensively studied around galaxies (Tumlinson et al. 2011), its geometrical distribution around galaxies, which can yield improved understanding of the origins of such gas, has not been thoroughly studied. Here we summarize work from Kacprzak et al. (2015) who examine the multi-phase inflow and outflow azimuthal distribution using Ovi absorption around galaxies.

\section{The Sample}

Our sample consists of 29 O vi absorbing $(\mathrm{EW}>0.1 \AA)$ and 24 non-absorbing $(\mathrm{EW}<0.1 \AA)$ galaxies with spectroscopically confirmed redshifts $(0.08<z<0.67)$ within $\sim 200 \mathrm{kpc}$ of bright background quasars. These galaxies are selected to be isolated such that there are no galaxies within $100 \mathrm{kpc}$ and having velocity separations less than $500 \mathrm{~km} \mathrm{~s}^{-1}$. The 


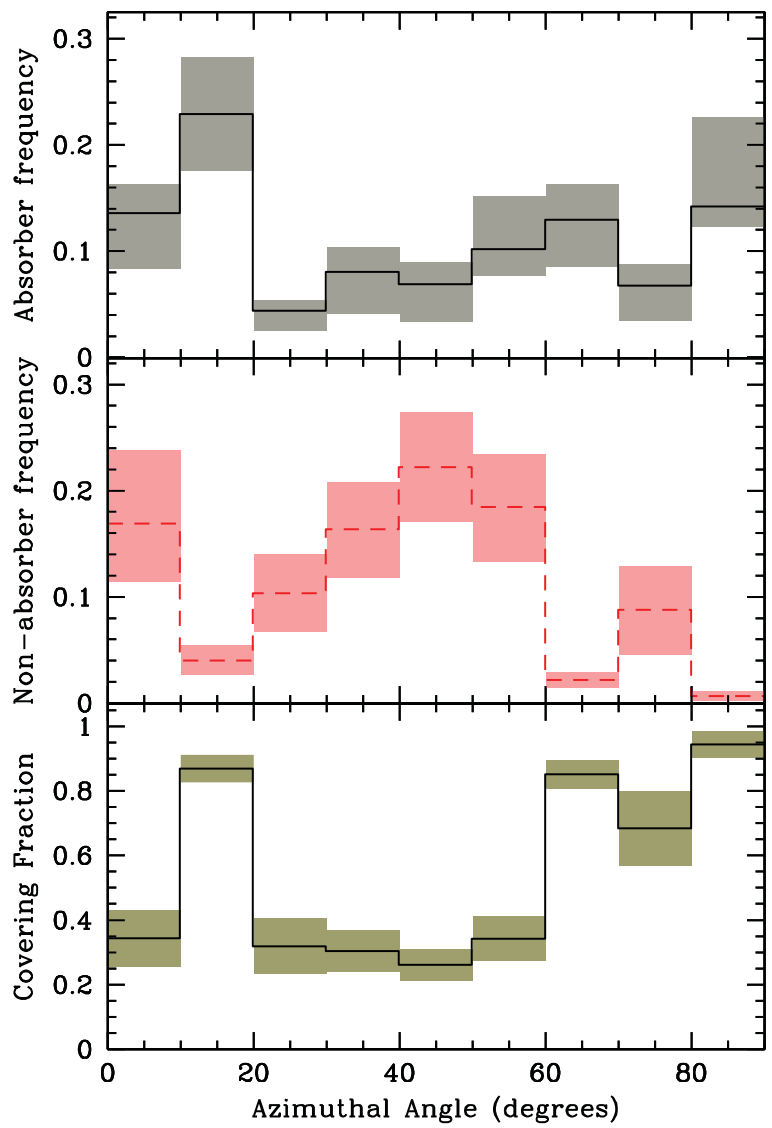

Figure 1. (Top) The azimuthal angle distribution for absorbing galaxies ( 0 degrees $\equiv$ along major axis and 90 degrees $\equiv$ along minor axis). The binned probability distribution functions are normalized such that the total area is equal to unity, providing an observed frequency in each bin. Shaded regions are $1 \sigma$ errors produced by bootstrapping the data. Absorption is detected with increased frequency towards the major and minor axes. (Middle) Azimuthal angle distribution for non-absorbing galaxies. Note the opposite effect, where non-absorbers peak where absorbers are near a minimum. (Bottom) The OVI gas covering fraction and $1 \sigma$ errors.

background quasars all have HST/COS and STIS spectra covering the OvI $\lambda \lambda 1031,1037$ doublet. The quasar fields all have high resolution HST WFPC-2, ACS, and WFC3 imaging. We used GIM2D (Simard et al. 2002) to model the galaxy morphological properties and the azimuthal angle relative to the galaxy projected major $\left(\Phi=0^{\circ}\right)$ and projected minor $\left(\Phi=90^{\circ}\right)$ axes and the quasar sight-line (for further details see Kacprzak et al. $2007 ; 2011)$.

\section{OVI Absorption as a Function of Galaxy Orientation}

Figure 1 shows the binned mean azimuthal angle probability distribution function (PDF) for the absorbing and non-absorbing galaxies. The shaded regions are the $1 \sigma$ deviations computed from bootstrapping the galaxy $\Phi$ distribution 10,000 times.

The presence of O VI absorption (EW $>0.1 \AA$ ) is azimuthally dependent and primarily occurs along the projected major axis within a half opening angle of $20^{\circ}$ and along the projected minor axis within a half opening angle of at least $30^{\circ}$. The slow rise of the 
absorber PDF and the sharp decline of the non-absorber PDF along the minor axis may suggest that OVI outflowing gas could occur within a half-opening angle as small as $30^{\circ}$ (from $60-90^{\circ}$ ) or even larger to $50^{\circ}$. The distribution of $\mathrm{OVI}$ is consistent with the expected geometry of major axis-fed inflows and minor axis-driven outflows and is consistent with previous results found for MgII absorption.

The frequency of non-detected O vi absorption $(\mathrm{EW}<0.1 \AA)$ as a function of azimuthal angle is highest between $30-60^{\circ}$, suggesting a paucity of detectable O vi absorbing gas at intermediate azimuthal angles. This suggests that OvI gas is not mixed throughout the CGM, but remains primarily within the outflowing winds and near to the planar disk region. We interpret the deficit of absorption and the surplus of non-absorbers within $\pm 10^{\circ}$ of the major axis possibly as colder gas residing in this area while more diffuse gas surrounds the cool dense as indicated by the peak at $20^{\circ}$.

In the bottom of Figure 1, we show the OVI covering fraction as a function of azimuthal angle. The covering fraction is highly dependent on $\Phi$ and is the highest along the projected major and minor axes. The azimuthal angle averaged covering fraction of our sample is $52 \%$. We see a deficit of absorption with a covering fraction of $35 \%$ within $10^{\circ}$ of the projected major axis. The covering fraction peaks at $85 \%$ at $20^{\circ}$ before dropping back to $35 \%$ and then rises again at a maximum (above $80 \%$ ) within $30^{\circ}$ of the projected minor axis.

We have further explored how the incidence of absorbers and non-absorbers depend on galaxy inclination and find that regardless of galaxy inclination, the absorbers have a preference for existing along the projected major and minor axes. The same is true for the non-absorbers, which continue to have a preference to exist at intermediate azimuthal angle. We present additional results in Kacprzak et al. (2015) showing how the Ovi azimuthal angle distribution is dependent on galaxy color and equivalent width.

\section{Summary}

The combined azimuthal results of Kacprzak et al. (2015) are consistent with current models of gaseous multi-phase outflows and accretion/recycling. However, it is still unclear whether we are still observing cold accretion filaments at redshifts $z<1$ or, the more likely scenario, we are tracing recycling of previously ejected gas. Obtaining absorption metallicities is the key in constraining the exact feedback processes that are occurring in and around galaxies.

\section{References}

Bordoloi, R., Lilly, S. J., Knobel, C., et al. 2011, ApJ, 743, 10

Bouché, N., Hohensee, W., Vargas, R., Kacprzak, G. G., et al. 2012, MNRAS, 426, 801

Kacprzak, G. G., Churchill, C. W., \& Nielsen, N. M. 2012, ApJ (Letters), 760, L7

Kacprzak, G. G., Churchill, C. W., Evans, J. L., Murphy, M. T., \& Steidel, C. C. 2011, MNRAS, 416,3118

Kacprzak, G. G., Churchill, C. W., Steidel, C. C., Murphy, M. T., \& Evans, J. L. 2007, ApJ, 662,909

Kacprzak, G. G., Muzahid, S., Churchill, C. W., Nielsen, N. M., \& Charlton, J. C. 2015, ApJ, 815,22

Lan, T.-W., Ménard, B., \& Zhu, G. 2014, ApJ, 795, 31

Simard, L., Willmer, C. N. A., Vogt, N. P., et al. 2002, ApJS, 142, 1

Tumlinson, J., Thom, C., Werk, J. K., et al. 2011, Science, 334, 948 